International Mathematical Forum, Vol. 9, 2014, no. 34, 1669 - 1672

HIKARI Ltd, www.m-hikari.com

http://dx.doi.org/10.12988/imf.2014.49165

\title{
A Generalization of the Inclusion-Exclusion Principle
}

\author{
Rafael Jakimczuk \\ División Matemática, Universidad Nacional de Luján \\ Buenos Aires, Argentina
}

Copyright (c) 2014 Rafael Jakimczuk. This is an open access article distributed under the Creative Commons Attribution License, which permits unrestricted use, distribution, and reproduction in any medium, provided the original work is properly cited.

\begin{abstract}
In this note we present a generalization of the inclusion-exclusion principle and an application of this more general principle.

Mathematics Subject Classification: 11A99

Keywords: Inclusion-exclusion principle

\section{Generalization of the Inclusion-Exclusion Prin- ciple}

The following theorem is sometimes called either the principle of cross-classification or the inclusion-exclusion principle. We now enunciate the principle.

Theorem 1.1 (Inclusion-exclusion principle)Let $S$ be a set of $N$ distinct elements, and let $S_{1}, \ldots, S_{r}$ be arbitrary subsets of $S$ containing $N_{1}, \ldots, N_{r}$ elements, respectively. For $1 \leq i<j<\ldots<l \leq r$, let $S_{i j \ldots l}$ be the intersection of $S_{i}, S_{j}, \ldots, S_{l}$ and let $N_{i j \ldots l}$ be the number of elements of $S_{i j \ldots l}$. Then the number $K$ of elements of $S$ not in any of $S_{1}, \ldots, S_{r}$ is

$$
K=N-\sum_{1 \leq i \leq r} N_{i}+\sum_{1 \leq i<j \leq r} N_{i j}-\sum_{1 \leq i<j<k \leq r} N_{i j k}+\ldots+(-1)^{r} N_{12 \ldots r}
$$

Proof. See, for example, [3] (page 84) or [2] (page 233). 
Theorem 1.2 Let $S$ be a set of $N$ distinct elements, that is

$$
S=\left\{a_{1}, a_{2}, \ldots, a_{N}\right\}
$$

and let $S_{1}, \ldots, S_{r}$ be arbitrary subsets of $S$ containing $N_{1}, \ldots, N_{r}$ elements, respectively. For $1 \leq i<j<\ldots<l \leq r$, let $S_{i j \ldots l}$ be the intersection of $S_{i}, S_{j}, \ldots, S_{l}$ and let $N_{i j \ldots l}$ be the number of elements of $S_{i j \ldots l}$. Let $f$ be a function with domain $S$ which assigns to each element $a_{m} \in S(m=1,2, \ldots, N)$ a complex number $f\left(a_{m}\right)(m=1,2, \ldots, N)$. Finally, let $T=S-\bigcup_{i=1}^{r} S_{i}$. The following formula holds.

$$
\begin{gathered}
\sum_{a_{m} \in T} f\left(a_{m}\right)=\sum_{a_{m} \in S} f\left(a_{m}\right)-\sum_{1 \leq i \leq r} \sum_{a_{m} \in S_{i}} f\left(a_{m}\right)+\sum_{1 \leq i<j \leq r} \sum_{a_{m} \in S_{i, j}} f\left(a_{m}\right) \\
-\sum_{1 \leq i<j<k \leq r} \sum_{a_{m} \in S_{i, j, k}} f\left(a_{m}\right)+\ldots+(-1)^{r} \sum_{a_{m} \in S_{1,2, \ldots, r}} f\left(a_{m}\right)
\end{gathered}
$$

Proof. The proof is similar as Theorem 1.1. See, for example, [3] (page 84) or [2] (page 233).

Remark 1.3 Note that if the function $f$ is the constant function $f\left(a_{m}\right)=$ $1(m=1,2, \ldots, N)$ then equation (2) becomes equation (1). That is, the inclusion-exclusion principle is a particular case of this more general principle, since

$$
\begin{aligned}
\sum_{a_{m} \in T} f\left(a_{m}\right) & =\sum_{a_{m} \in T} 1=K \\
\sum_{a_{m} \in S} f\left(a_{m}\right) & =\sum_{a_{m} \in S} 1=N \\
\sum_{a_{m} \in S_{i}} f\left(a_{m}\right) & =\sum_{a_{m} \in S_{i}} 1=N_{i}
\end{aligned}
$$

etc

\section{An Application}

If $n \geq 1$ the Euler's function $\varphi(n)$ is defined to be the number of positive integers not exceeding $n$ which are relatively prime to $n$. The following formula is well-known

$$
\varphi(n)=n \prod_{p \mid n}\left(1-\frac{1}{p}\right)
$$

where, the product run on the different prime divisors $p$ of $n$. In this note $p$ denotes a positive prime. 
Let us consider the sum $1^{s}+2^{s}+\cdots+n^{s}$ where $s$ is a arbitrary but fixed positive integer. It is well known there exists a polynomial in $n$ of degree $s+1$ and rational coefficients such that

$$
1^{s}+2^{s}+\cdots+n^{s}=c_{s+1} n^{s+1}+c_{s} n^{s}+c_{s-1} n^{s-1}+c_{s-2} n^{s-2}+\cdots+c_{1} n
$$

For example, we have

$$
\begin{gathered}
1+2+\cdots+n=\frac{1}{2} n^{2}+\frac{1}{2} n \\
1^{2}+2^{2}+\cdots+n^{2}=\frac{1}{3} n^{3}+\frac{1}{2} n^{2}+\frac{1}{6} n \\
1^{3}+2^{3}+\cdots+n^{3}=\frac{1}{4} n^{4}+\frac{1}{2} n^{3}+\frac{1}{4} n^{2}
\end{gathered}
$$

There exist various methods in the literature to obtain these polynomials.

Theorem 2.1 Let $\varphi_{s}(n)$ denote the sum of the sth powers of the numbers $\leq n$ and relatively prime to $n$. The following formula holds (see (3) and (4))

$$
\begin{aligned}
\varphi_{s}(n) & =c_{s+1} n^{s} \varphi(n)+\prod_{p \mid n}(1-p) c_{s-1} n^{s-1}+\prod_{p \mid n}\left(1-p^{2}\right) c_{s-2} n^{s-2} \\
& +\cdots+\prod_{p \mid n}\left(1-p^{s-1}\right) c_{1} n
\end{aligned}
$$

Proof. We have (see (4))(The $p_{i}$ are the different prime divisors of $n$ )

$$
\begin{gathered}
\left(p_{i} 1\right)^{s}+\left(p_{i} 2\right)^{s}+\cdots+\left(p_{i} \frac{n}{p_{i}}\right)^{s}=p_{i}^{s}\left(1^{s}+2^{s}+\cdots+\left(\frac{n}{p_{i}}\right)^{s}\right) \\
=c_{s+1} \frac{n^{s+1}}{p_{i}}+c_{s} n^{s}+p_{i} c_{s-1} n^{s-1}+p_{i}^{2} c_{s-2} n^{s-2}+\cdots+p_{i}^{s-1} c_{1} n \\
\left(p_{i} p_{j} 1\right)^{s}+\left(p_{i} p j 2\right)^{s}+\cdots+\left(p_{i} p_{j} \frac{n}{p_{i} p_{j}}\right)^{s}=p_{i}^{s} p_{j}^{s}\left(1^{s}+2^{s}+\cdots+\left(\frac{n}{p_{i} p_{j}}\right)^{s}\right) \\
=c_{s+1} \frac{n^{s+1}}{p_{i} p_{j}}+c_{s} n^{s}+p_{i} p_{j} c_{s-1} n^{s-1}+p_{i}^{2} p_{j}^{2} c_{s-2} n^{s-2}+\cdots+p_{i}^{s-1} p_{j}^{s-1} c_{1} n
\end{gathered}
$$

etc

Substituting (4), (9), (10), etc into (2) we obtain (8). The theorem is proved. 
Using formulae (5), (6) and (7) we obtain the following examples of Theorem 2.1 .

$$
\begin{gathered}
\varphi_{1}(n)=\frac{1}{2} n \varphi(n) \\
\varphi_{2}(n)=\frac{1}{3} n^{2} \varphi(n)+\prod_{p \mid n}(1-p) \frac{1}{6} n \\
\varphi_{3}(n)=\frac{1}{4} n^{3} \varphi(n)+\prod_{p \mid n}(1-p) \frac{1}{4} n^{2}
\end{gathered}
$$

Other proof of Theorem 2.1 using a different method is given in [1, chapter 2].

ACKNOWLEDGEMENTS. The author is very grateful to Universidad Nacional de Luján.

\section{References}

[1] T. M. Apostol, An Introduction to Analytic Number Theory, Springer, 1976. http://dx.doi.org/10.1007/978-3-662-28579-4

[2] G. H. Hardy and E. M. Wright, An Introduction to The Theory of Numbers, Oxford, 1960.

[3] W. J. LeVeque, Topics in Number Theory, Volume 1, Addison-Wesley, 1958.

Received: September 15, 2014; Published: November 12, 2014 